\title{
Recent Developments in Selected Areas of Research Initiated or Significantly Advanced by Bernhard Gross
}

Bernhard Gross has been and continues to be a great inspiration to his many friends and colleagues all over the world. Therefore, it is no wonder that they wish to thank him again and again for the ideas, the leadership, the encouragement, and the support he has given them over several decades of this century. Consequently, a call was sent out to several of his closest scientific friends in order to assemble a series of short invited reviews which permit a glimpse at some of the rich and wide-spread physics that originated from Bernhard Gross' many seminal contributions. For several reasons, the present special issue became rather a bouquet of various flowers coming directly or indirectly from his garden than a systematical treatise of the many plants that Bernhard Gross helped sow and grow. We hope that the present collection will nevertheless be of some use to the physics community. (in spite of its many shortcomings and omissions for which the editors alone are responsible).

We are deeply grateful to all the colleagues who found the time to write a suitable contribution and to help with the refereeing for this special issue. Special thanks are due to Sergio Mascarenhas and Gerhard Sessler for offering accounts of Bernhard Gross' many contributions to physics in Brazil and in general. Most of all, we wish to thank Bernhard Gross for the example and the friendship he has always provided for us, and also for his original scientific contribution to this issue which is, as usually, up to date, novel and inspiring. We hope that he will accept this collection of papers as a small token of our appreciation for him.

In order to facilitate the use of this special issue, its twelve contributions have been divided into three groups of four papers each.

The first group is dedicated to radiation effects and appropriately begins with an original report by Bernhard Gross and his recent coworkers about electric-field effects on the electron emission from dielectrics during irradiation with only slightly penetrating electrons. It is followed by original and review articles on the experimental investigation and the theoretical modelling of charge dynamics during and after electron irradiation of polymer films, on the state of the art in the field of insulating spacecraft materials under high-energy irradiation, and on the investigation and application of specifically chosen materials for the temporary storage of $\mathrm{x}$-ray images. All four papers deal in one way or another with the effects of radiation on the electrical properties of dielectric materials, a topic to which Prof. Gross made his first seminal contributions in the 1950s. In the second group, papers on charge storage and dipole polarization are assembled. The first paper comes directly from the Gross school in São Carlos and deals with constant-current charging by means of the corona triode which was pioneered by Prof. Gross in the 1970s. In the following three surveys, space-charge investigations on polymers for high-voltage insulation, the evidence for an interaction of charges and dipoles in the ferroelectric polymer PVDF, and the preparation and application of non-uniformly poled polymer electrets are briefly summarized. All four papers touch upon the concept of homoand heterocharge introduced by Prof. Gross in the 1940s. The same concept is also present in the first contribution of the third group containing papers on dielectrics and conduction, but this time with emphasis on the influence of molecular dipoles on charge storage and transport. Two theoretical studies on the discrimination between dielectric relaxation and conduction and on time-dependent conduction in disordered solids follow. Last, but not least, two members of the Gross school in São Carlos report about recent work on semiconducting polymers and their applications. Again, all four papers deal with topics close to Prof. Gross' early advances in the related fields of dielectric relaxation and of charge transport in insulating materials. 
Not surprisingly, this collection contains four papers from Brazil, the country Gross made his home in 1933, as well as four papers from Germany, his country of origin to which he returned several times since the end of the Second World War. Two contributions come from the United States where Gross spent several very fruitful visits and two from Australia and Poland, but these are also motivated by contacts mainly established in the United States and in Brazil, respectively. All contributions are more or less closely related to the theme of electrets, i.e. of dielectrics with quasi-permanent charge storage and/or dipole orientation, a field of physics which continues to be one of the leitmotifs of Bernhard Gross' life and which he inspired so much.

Reimund Gerhard-Multhaupt

Institut für Physik der Universität Potsdam

Am Neuen Palais 10, D-14469 Potsdam, Germany

Guilherme F. Leal Ferreira and Roberto M. Faria

Instituto de Física de São Carlos, Universidade de São Paulo

Caixa Postal 369, CEP 13560-970 São Carlos, SP, Brazil 